\title{
Ultrasonographic measurement of subclavian vein diameter and regression modeling in pediatric patients from a single Korean facility
}

\author{
Min Hye Oh, Woo Suk Chung, Yo Han Kim, Byung Muk Kim, and Sang-Il Park \\ Department of Anesthesiology and Pain Medicine, Chungnam National University Hospital, Daejeon, Korea
}

Central venous catheterization (CVC) is vital for anesthetic management in pediatric patients undergoing major surgery [1]. The internal jugular vein (IJV), subclavian vein (SCV), and femoral vein are the most commonly used sites for CVC. Unfortunately, the failure rate and incidence of complications are much higher in pediatric patients due to the relatively small size of the vessels [2]. Experience is essential in order to successfully perform CVC in pediatric patients without causing complications. Other significant factors include the selection of an appropriate vein and a catheter of a suitable size. The IJV is relatively larger and easier to access than the SCV, and has been preferred for CVC in pediatric patients by many physicians. Yet, the IJV is easily collapsed by the ultrasound (US) probe or by needle pressure, making CVC challenging even though the vein may be well observed via US. In contrast, the SCV is not easily collapsed due to the presence of the clavicle and can be more appropriate when long-term catheter placement is required [3]. Although there are several studies regarding the size of the IJV in pediatric patients $[1,2]$, few studies have been performed related to the size of the SCV. We attempted to find the most suitable variable, such as age, weight, or height, that could predict the size of the SCV in pediatric patients by measuring the size of the SCV in a group of these patients using ultrasound.

Thirty-eight pediatric patients from 1 month to 10 years of age, who underwent an elective surgery were included in this study. Patients included in the study were scheduled for minor operations: inguinal hernia repair, hydrocelectomy, circumci- sion, and upper and lower extremity surgeries. Patients with congenital or cardiovascular diseases, and patients scheduled for cardiac, intracranial, or major vascular operations were excluded from the study. After induction of general anesthesia, patients were placed in a supine position with their arms positioned next to the trunk. The SCV was located with a linear probe using a method implemented previously [4]. Briefly, the longitudinalsection image of the SCV was obtained with an $18 \mathrm{MHz}$ linear probe (LA435: 6-18 MHz, Esaote, Genova, Italy). Images were obtained by a single physican after acquiring a view in which the SCV seemed to have the largest diameter and the clavicle was positioned in the center of the image. Images were obtained three times on both sides at the end-expiratory phase of ventilation and were measured by two different study participants using image analysis software (MyLab ${ }^{\mathrm{TM}}$ Desk, Esaote, Genova, Italy). The internal diameter of the SCV was measured directly laterally and medially to the clavicle shadow, and defined as the average of these two values. The average of the values measured by the two different study participants was used as the SCV diameter in each pediatric patient.

For statistical analysis, variables of regression modeling were selected using the stepwise selection method. R 3.1.0 (R Core Team, Vienna, Austria) was used as the statistical software, and variables were selected using the faraway and leaps packages. The leaps package automatically selects variables, and the Bayesian information criterion was used as the selection criterion. Sex, height, weight, and age were selected as independent variables

Corresponding author: Sang-Il Park, M.D., Department of Anesthesiology and Pain Medicine, Chungnam National University Hospital, 282, Munhwa-ro, Jung-gu, Daejeon 301-721, Korea. Tel: 82-42-280-7840, Fax: 82-42-280-7968, E-mail: goodlebang@gmail.com

(c) This is an open-access article distributed under the terms of the Creative Commons Attribution Non-Commercial License (http:// creativecommons.org/licenses/by-nc/3.0/), which permits unrestricted non-commercial use, distribution, and reproduction in any medium, provided the original work is properly cited. 
A

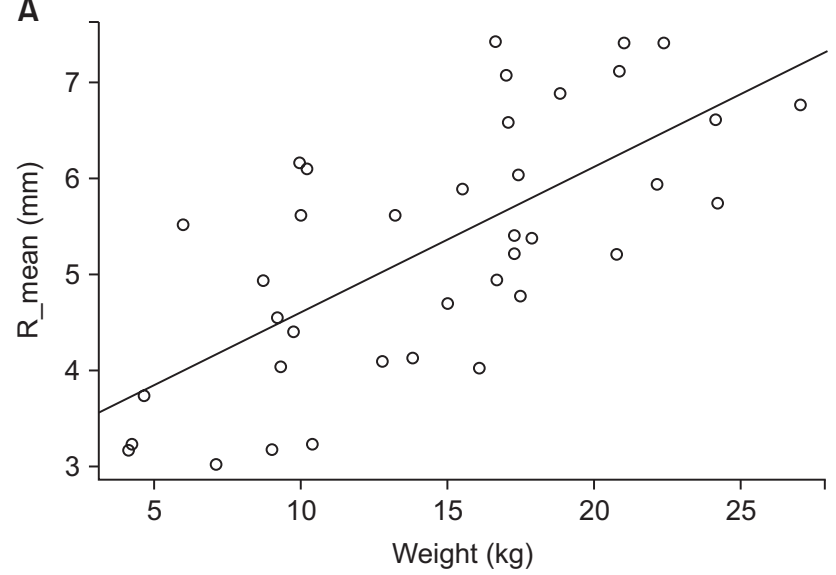

B

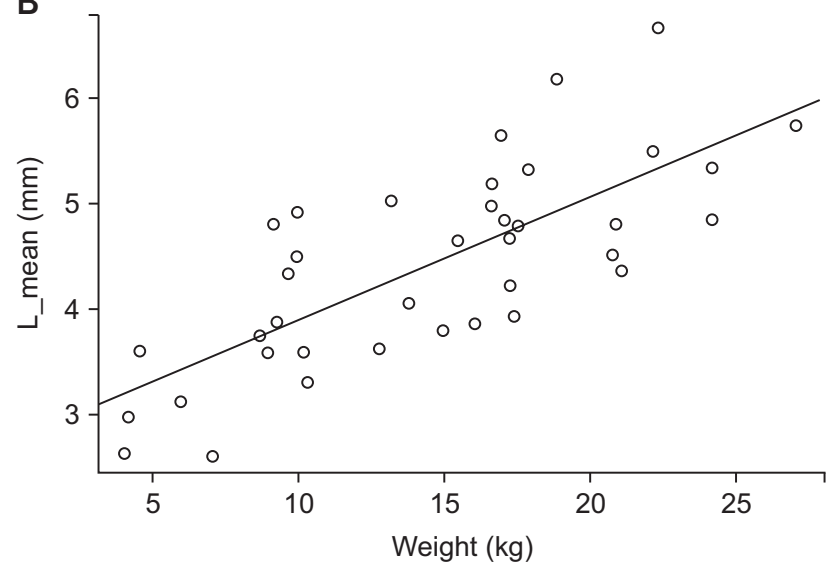

Fig. 1. (A) Correlation between weight and right SCV diameter. Equation of regression line: right SCV diameter $(\mathrm{mm})=3.09+0.15 \times$ weight $(\mathrm{kg})$. Number of patients: $n=38$. (B) Correlation between weight and left SCV diameter. Equation of regression line: left SCV diameter $(\mathrm{mm})=2.73+0.12$ $\times$ weight $(\mathrm{kg})$. Number of patients: $\mathrm{n}=38$.

for modeling, and variables with a $\mathrm{P}$ value less than 0.05 in the result were chosen. An independent t-test was conducted with continuous variables, and a paired t-test was used for the statistical significance in the difference of the SCV diameter measured by the two examiners. The study was conducted from March to October, 2013. During this period, 39 pediatric patients were measured and one pediatric patient was excluded from the analysis due to the omission of height. For the diameter of each SCV, the values measured by examiners 1 and 2 were respectively 5.29 $\pm 1.34 \mathrm{~mm}$ and $5.32 \pm 1.31 \mathrm{~mm}$ in the right SCV and $3.72 \pm 0.79$ $\mathrm{mm}$ and $5.13 \pm 1.13 \mathrm{~mm}$ in the left SCV. The diameter of each SCV was defined as the average of the measurements by the two examiners. Results from the statistical software on variable selection showed that weight had the strongest correlation with SCV diameter. The results of regression modeling of the right SCV diameter were: intercept $(3.09 \pm 0.41)$, slope of weight $(0.15 \pm 0.03$, $\mathrm{P}$ value $=0.00)$, and $\mathrm{R}^{2}(0.48)$. The results of regression modeling of the left SCV diameter were: intercept $(2.73 \pm 0.27)$, slope of weight $(0.12 \pm 0.02$, P value $=0.00)$, and $\mathrm{R}^{2}(0.56)$ (Fig. 1$)$. Con- sistent with our results, a previous study which compared the sizes of the IJV and SCV in small infants weighing less than 4.6 $\mathrm{kg}$ also found that the size had a strong correlation with weight [5]. Sayin et al. [1] reported that the IJV diameter in 1-monthold to 15-year-old patients correlated with their age, height, weight, and head circumference. In addition, Karazincir et al. [2] reported that the dimensions of the IJV in Turkish children aged 0 to 6 correlated with age, weight and height. However, none of these studies compared the correlation degree among variables, as was done in our present study.

In conclusion, our study is the first to measure the size of the SCV in Korean pediatric patients, analyze the measured values and develop a model that can predict the diameter of the SCV. Our results suggest that weight is the variable most appropriate for predicting the pediatric patients' SCV diameter when estimated with a regression equation. However, further studies with a larger population are needed in order to build a more precise model for predicting SCV diameter.

\section{References}

1. Sayin MM, Mercan A, Koner O, Ture H, Celebi S, Sozubir S, et al. Internal jugular vein diameter in pediatric patients: are the J-shaped guidewire diameters bigger than internal jugular vein? An evaluation with ultrasound. Paediatr Anaesth 2008; 18: 745-51.

2. Karazincir S, Akoğlu E, Balci A, Sangün O, Okuyucu S, Ozbakiş C, et al. Dimensions of internal jugular veins in Turkish children aged between 0 and 6 years in resting state and during Valsalva maneuver. Int J Pediatr Otorhinolaryngol 2007; 71: 1247-50.

3. Rhondali O, Attof R, Combet S, Chassard D, de Queiroz Siqueira M. Ultrasound-guided subclavian vein cannulation in infants: supraclavicular approach. Paediatr Anaesth 2011; 21: 1136-41.

4. Park SI, Kim YH, So SY, Kim MJ, Kim HJ, Kim JK. Ultrasound-guided subclavian catheterization in pediatric patients with a linear probe: a case series. Korean J Anesthesiol 2013; 64: 541-4.

5. Breschan C, Platzer M, Jost R, Stettner H, Likar R. Size of internal jugular vs subclavian vein in small infants: an observational, anatomical evaluation with ultrasound. Br J Anaesth 2010; 105: 179-84. 